\title{
Quercetin in Tartary Buckwheat Induces Autophagy against Protein Aggregations
}

\author{
Sumiko Ikari ${ }^{1}$, Qiang Yang ${ }^{2}$, Shiou-Ling Lu ${ }^{1}$, Yuancai Liu ${ }^{2}$, Feike Hao ${ }^{1}$, Guoqiang Tong ${ }^{3}$, Shiguang Lu ${ }^{3}$ \\ and Takeshi Noda ${ }^{1, *(D)}$ \\ 1 Center for Frontier of Oral Sciences, Graduate School of Dentistry, Osaka University, Osaka 5650871, Japan; \\ sikari@kuhp.kyoto-u.ac.jp (S.I.); sally711017@dent.osaka-u.ac.jp (S.-L.L.); haofeike@outlook.com (F.H.) \\ 2 Hubei Provincial Key Lab for Quality and Safety of Traditional Chinese Medicine Health Food, \\ Daye 435100, China; yq@jingpai.com (Q.Y.); lyc@jingpai.com (Y.L.) \\ 3 Jing Brand Company, Ltd., Daye 435100, China; winebag@jingpai.com (G.T.); lushiguang@jingpai.com (S.L.) \\ * Correspondence: takenoda@dent.osaka-u.ac.jp; Tel.: +81-6-6879-2976
}

Citation: Ikari, S.; Yang, Q.; Lu, S.-L.; Liu, Y.; Hao, F.; Tong, G.; Lu, S.; Noda, T. Quercetin in Tartary Buckwheat Induces Autophagy against Protein Aggregations. Antioxidants 2021, 10, 1217. https:// doi.org/10.3390/antiox10081217

Academic Editor: Elzbieta Janda and María P. Portillo

Received: 8 June 2021

Accepted: 25 July 2021

Published: 29 July 2021

Publisher's Note: MDPI stays neutral with regard to jurisdictional claims in published maps and institutional affiliations.

Copyright: (c) 2021 by the authors. Licensee MDPI, Basel, Switzerland. This article is an open access article distributed under the terms and conditions of the Creative Commons Attribution (CC BY) license (https:/ / creativecommons.org/licenses/by/ $4.0 /)$.

\begin{abstract}
Tartary buckwheat is used as an ingredient in flour and tea, as well as in traditional Chinese medicine for its antioxidant effects. Here, we found that an ethanol extract of tartary buckwheat (TBE) potently induced autophagy flux in HeLa cells by suppressing mTORC1 activity, as revealed by dephosphorylation of the mTORC1 substrates Ulk1, S6K, and 4EBP, as well as by the nuclear translocation of transcriptional factor EB. In addition to non-selective bulk autophagy, TBE also induced aggrephagy, which is defined as autophagy against aggregated proteins. Quercetin is a flavonol found at high levels in TBE. We showed that quercetin induced both non-selective bulk autophagy and aggrephagy. These effects were also observed in Huh-7 cells derived from hepatocytes. Thus, aggrephagy induction by TBE and quercetin may relieve alcoholic hepatitis, which is closely linked to the accumulation of protein aggregations called Mallory-Denk bodies.
\end{abstract}

Keywords: tartary buckwheat; mTORC1; aggrephagy

\section{Introduction}

Macroautophagy (hereafter referred to simply as autophagy) is an intracellular degradation process that supports cellular homeostasis [1]. The mechanisms that underlie autophagy were initially characterized in yeast studies, but are now understood in mammals as well [2]. As a result, increasing numbers of studies have demonstrated the vital connection between autophagy and a wide variety of human diseases, such as cancer and neurodegenerative diseases [3]. To address these autophagy-related conditions, a number of attempts by the pharmaceutical industry and academia are underway worldwide to artificially regulate autophagy activity in vivo by the use of exogenous compounds [4]. In particular, traditional Chinese medicine has attracted wide attention as a source of potential autophagy modulators [5]. This therapeutic approach has a different foundation than European medicine, and it is broadly accepted, mainly in East Asia. It is based on a long history of prescription and experience, and therefore while its physiological effects are assured to some degree, the underlying molecular mechanisms are largely undetermined. We recently reported that Shigyakusan, a mixture of compounds used in Kampo (Japanese-style traditional Chinese medicine) and a close relative of Shini-san in traditional Chinese medicine, has a suppressive effect on autophagy [6].

Tartary buckwheat (Fagopyrum tataricum) is a species of herbaceous plant whose grain is used to prepare flour, noodles, and herbal tea, all mainly in Central and East Asia [7]. Compared to common buckwheat (Fagopyrum esculentum), it has a characteristically bitter flavor. In addition to its use in food, the ethanol extract of tartary buckwheat (TBE) is utilized as a component of traditional Chinese medicine [8]. The administration of TBE to mouse brain and liver was shown to reduce antioxidant enzyme levels [8]. It also exerted 
an antibacterial effect against Propionibacterium and Staphylococci species [9]. We conducted a pilot survey to determine whether any of the ingredients of Maopu buckwheat liquor, a Chinese liquor containing various Chinese herbal medicine extracts, affected autophagy activity, and found that TBE strongly induced autophagy. Therefore, we performed the present study to elucidate the molecular mechanisms of this property of TBE and its effect on hepatocytes.

\section{Materials and Methods}

\subsection{Preparation of Tartary Buckwheat Extract}

Tartary buckwheat grain powder was provided by Jing Brand Company, Ltd., in Hubei, China. Briefly, tartary buckwheat grain was boiled in 60\% $(v / v)$ ethanol and subjected to centrifugation, and the supernatant was concentrated into powder by vacuum drying centrifugation. The provided power was dissolved in $42 \%(v / v)$ ethanol, heated at $95{ }^{\circ} \mathrm{C}$ for $10 \mathrm{~min}$, and then ultra-centrifuged at $100,000 \times g$ for $60 \mathrm{~min}$ at $23{ }^{\circ} \mathrm{C}$. The supernatant, which was stored at $-20^{\circ} \mathrm{C}$, was used as TBE.

\subsection{Antibodies}

The following antibodies were used at the indicated dilution ratios for immunoblotting and immunostaining assays: rabbit anti-phospho-ULK1 (Ser757) (6888S, Cell Signaling, MA, USA), 1/1000; mouse anti-ULK1 (C1918, Santa Cruz Biotechnology, TX, USA), 1/1000; rabbit anti-transcriptional factor EB (TFEB) (4240S, Cell Signaling, MA, USA), 1/2000; mouse anti-p70S6 kinase (49D7, 2708S, Cell Signaling, MA, USA), 1/1000; rabbit anti-4EBP1 (9452S, Cell Signaling, MA, USA), 1/1000; mouse anti-tubulin (T9026, Sigma-Aldrich, St. Louis, MI, USA), 1/10000; rabbit anti-p62 (SQSTM1) (PM045, MBL), 1/1000; rabbit antiLC3 (PM036, MBL, Tokyo, Japan), 1/1000 (immunoblotting) or 1/500 (immunostaining); HRP-conjugated anti-rabbit IgG secondary antibody (7074S, Cell Signaling, MA, USA), 1/10000; HRP-conjugated anti-mouse IgG secondary antibody (1031-05, Southern Biotech, Birmingham, AL, USA), 1/10000; and Alexa Fluor Plus 488 goat anti-rabbit secondary antibody (A32731, Invitrogen, MA, USA), 1/1000.

\subsection{Cell Culture}

HeLa and Huh7 cells were cultured in Dulbecco's modified Eagle's medium (DMEM) (D6429, Sigma-Aldrich, St. Louis, MI, USA) with 10\% FBS (F7524, Sigma-Aldrich, St. Louis, MI, USA) and incubated in $5 \% \mathrm{CO}_{2}$ at $37^{\circ} \mathrm{C}$. HeLa cells stably expressing ULK1-EGFP, GFPAtg5, GFP-WIPI, tf-LC3, and GFP-TFEB were described previously [6]. For performing LC3 flux assays, 125 nM bafilomycin A1 (023-11641, Wako, Osaka, Japan) was used. For the aggrephagy experiment, cells were treated with $5 \mu \mathrm{g} / \mathrm{mL}$ puromycin (160-23151, Lot PTL1732, Wako, Osaka, Japan) for $4 \mathrm{~h}$ and then washed with new medium. Torin-1 (475991, Calbiochem, Darmstadt, Germany) was used at a concentration of 250 nM. Rutin (30319-04, Nacalai tesque, Kyoto, Japan) and was dissolved in 42\% ethanol. Quercetin (10005169, Cayman, MI, USA) was first dissolved in DMSO at a concentration of $210 \mathrm{mM}$ and then dissolved with $42 \%$ ethanol.

\subsection{MTT (Methylthialazole Tetrazolium) Assay for Cell Death}

Cells were dispensed into 96-well microplates at $5 \times 10^{4}$ cells in $200 \mu \mathrm{L}$ per well. After overnight culture, cells were treated with the indicated concentrations of TBE for an additional $48 \mathrm{~h}$. Cells were washed in DMEM with $10 \%$ FBS and then incubated with $5 \mu \mathrm{g} / \mathrm{mL}$ MTT (345-01821, Dojindo, Tokyo, Japan) in $100 \mu \mathrm{L}$ medium for $2 \mathrm{~h}$. To lyse MTTgenerated crystals, $100 \mu \mathrm{L}$ of $10 \%$ sodium dodecyl sulfate was added after the removal of the MTT medium, and the solution was incubated at $37^{\circ} \mathrm{C}$. Twenty hours later, plates were assessed by spectrophotometry at $570 \mathrm{~nm}$.

\subsection{Western Blotting}

Sample preparation and western blotting were performed, as previously described [6]. 


\subsection{Immunofluorescence and Confocal Microscope Observation}

Immunofluorescence and confocal microscope observation were performed, as previously described [6]. Images were acquired using a TCS SP8 confocal laser scanning fluorescence microscope (Leica, Wetzlar, Germany) equipped with an objective lens (HC PL APO 63x/1.40 OIL CS2, Leica). The number of fluorescent punctae was determined using ImageJ based on the size threshold specified in each legend. For the analysis of green fluorescent protein (GFP)-TFEB, the fluorescence intensities of certain areas in cytoplasmic and nuclear regions were measured using ImageJ.

\section{Results}

\subsection{TBE Induces Autophagy}

We first performed a pilot investigation using tf-LC3 (tandem fluorescent (GFP-RFP) LC3 protein) in HeLa cells to ascertain whether any of the five ingredients of Maopu buckwheat liquor affect autophagy activity [10]. We discovered that a $42 \%(v / v)$ ethanol extract of TBE induced autophagy. To determine how cell viability was affected by using ethanol as a solvent, HeLa cells were treated with a series of ethanol concentrations from 0 to $10 \%(v / v)$ for $24 \mathrm{~h}$ and subjected to an MTT assay. The highest ethanol concentration obtained without affecting cellular viability was 2\% $(v / v)$ (Supplementary Figure S1A). Next, we performed MTT assays with a series of concentrations of TBE dissolved in ethanol, and confirmed that using a concentration of $1500 \mu \mathrm{g} / \mathrm{mL}$ for $6 \mathrm{~h}$ did not affect cellular viability (Supplementary Figure S1B). Therefore, we adopted $1500 \mu \mathrm{g} / \mathrm{mL}$ of TBE dissolved in $2 \%$ ethanol for further analyses.

TBE treatment increased the formation of GFP-positive punctae, which represented autophagosomes or isolation membranes (Figure 1A) [11]. RFP-positive punctae were also observed, representing autophagosomes, isolation membranes, or autolysosomes (Figure 1A) [12]. The decrease in the ratio of the GFP/RFP signal intensity indicated that autophagosomes or isolation membranes had disintegrated during autophagy. Torin-1, a well-established inducer of autophagy, decreased the ratio of the GFP/RFP signal intensity (Figure 1B) [13]. Likewise, TBE decreased the GFP/RFP signal intensity in comparison to vehicle control (Figure 1B).

A

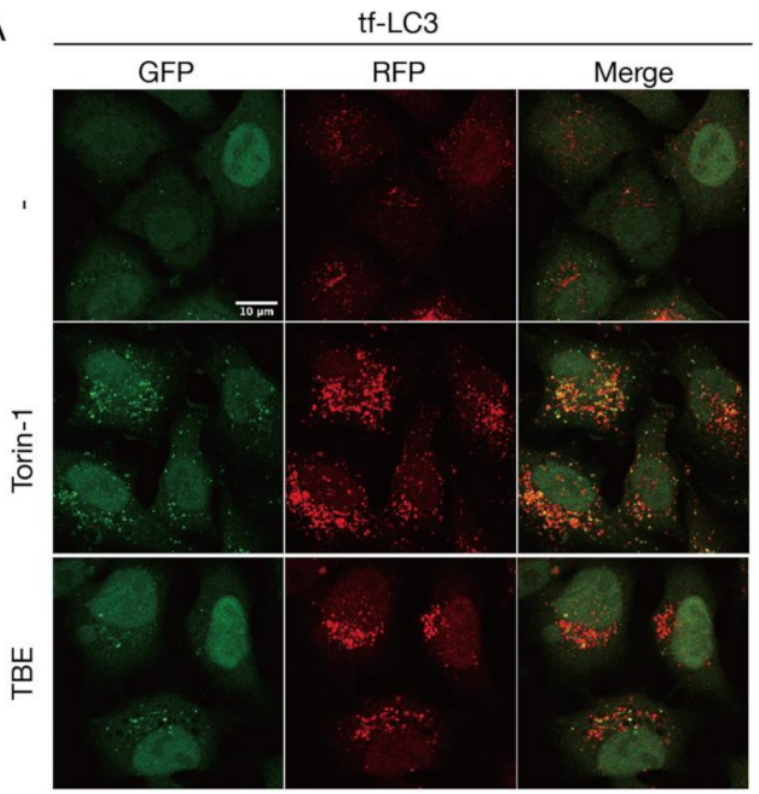

B

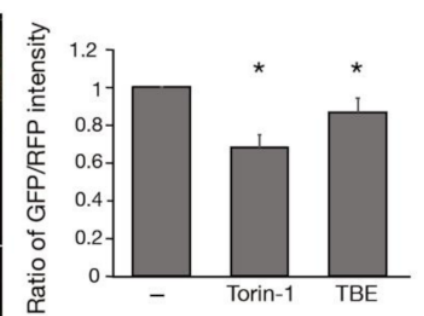

Figure 1. TBE induces autophagy progression. (A) Tf-LC3-expressing HeLa cells were treated with or without Torin-1 or TBE for $6 \mathrm{~h}$ under nutrient-rich conditions. Bar:10 $\mu \mathrm{m}$. (B) The graph shows the signal intensity ratio of GFP/RFP in each field of view after $6 \mathrm{~h}$. Median: line; upper and lower quartiles: boxes; 1.5 interquartile range: whiskers. ${ }^{*}$ denotes $p<0.05$ by unpaired two-tailed Student's $t$-test. 
We next assessed TBE-induced autophagy activity using a flux assay based on Western blotting of the autophagy marker LC3 [14]. Degradation of the LC3-II form, which is covalently conjugated to phosphatidylethanolamine, occurs upon fusion of autophagosomes with lysosomes. The extent of autophagy induction can be assessed by inhibiting this degradation by treatment with bafilomycin A1, a lysosomal acidification inhibitor. HeLa cells were treated with TBE for $6 \mathrm{~h}$ with or without bafilomycin A1, along with vehicle only (DMEM) or Torin-1 as controls (Figure 2). The results showed that LC3-II accumulated in samples treated with either TBE or Torin-1, indicating the induction of autophagy.
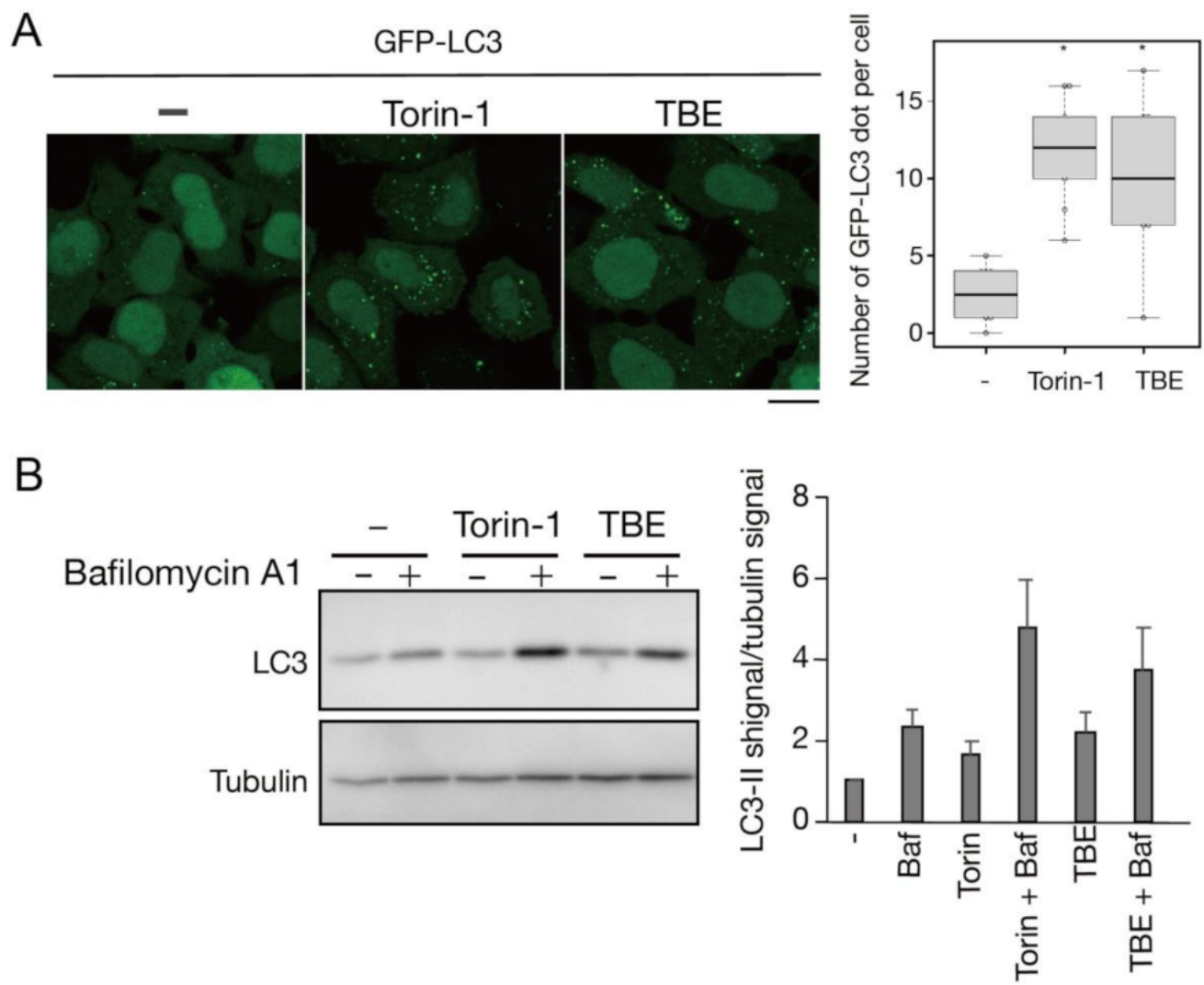

Figure 2. TBE induces autophagy flux under nutrient-rich conditions. (A) GFP-LC3-expressing HeLa cells were treated with Torin-1 or TBE for $6 \mathrm{~h}$ under nutrient-rich conditions. The number of GFP punctae per cell was counted, with over 20 cells in each sample. Median: line; upper and lower quartiles: boxes; 1.5 interquartile range: whiskers. Bar: $10 \mu \mathrm{m}(\mathbf{B})$ HeLa cells were treated with Torin-1 or TBE with or without bafilomycin A1 for $6 \mathrm{~h}$. The lysates were assessed by Western blotting with anti-LC3 antibody. The graph shows the average and standard deviation of the ratio of the LC3 signal to the tubulin signal from three independent experiments. ${ }^{*}$ denotes $p<0.05$ by unpaired two-tailed Student's $t$-test.

\subsection{TBE Induces Autophagosome Formation}

We next examined whether TBE affected autophagosome formation. Atg5 is a protein involved in the autophagosome formation process; it associates with isolation membranes and is then released from these membranes after autophagosome formation is complete [15]. Therefore, Atg5-positive structures represent isolation membranes/phagophores. TBE treatment increased GFP-Atg5-positive puncta structures in HeLa cells even under nutrient-rich conditions, indicating the formation of autophagosomes (Figure 3A). ULK1 is a protein kinase that comprises a protein complex along with FIP200/RB1CC1, Atg101, and Atg13; this complex constitutes a scaffold for autophagosome formation [16]. TBE treatment induced ULK1-EGFP puncta signals under nutrient-rich conditions (Figure 3B). WIPI1 is another core protein that is recruited to isolation membranes/phagophores and autophagosomes 
as a result of its binding affinity for phosphatidylinositol 3-phosphate [17]. The number of GFP-WIPI1 punctae was increased by TBE treatment (Figure 3C). Collectively, these data indicate that TBE induces autophagy in the step before autophagosome formation.

A

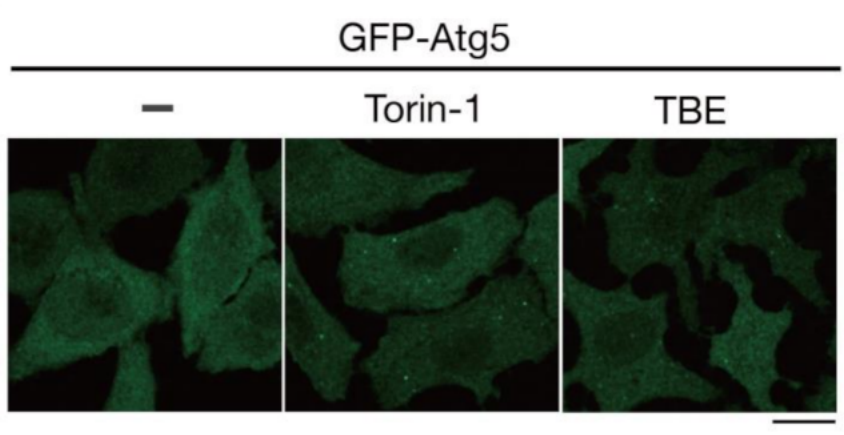

B

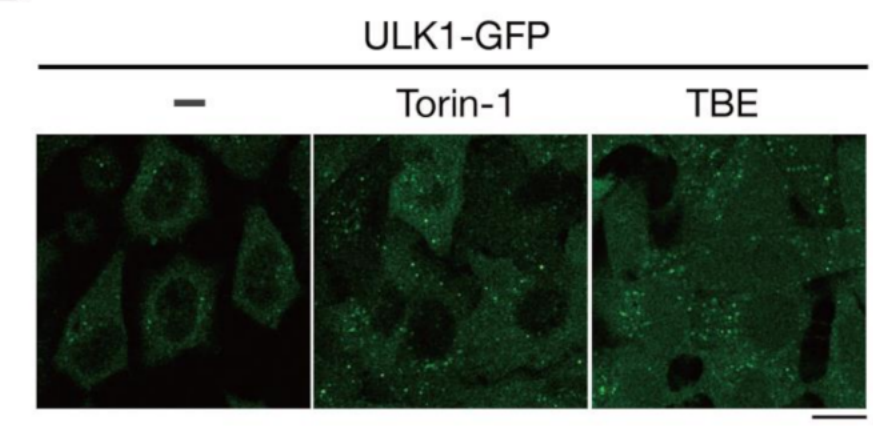

C

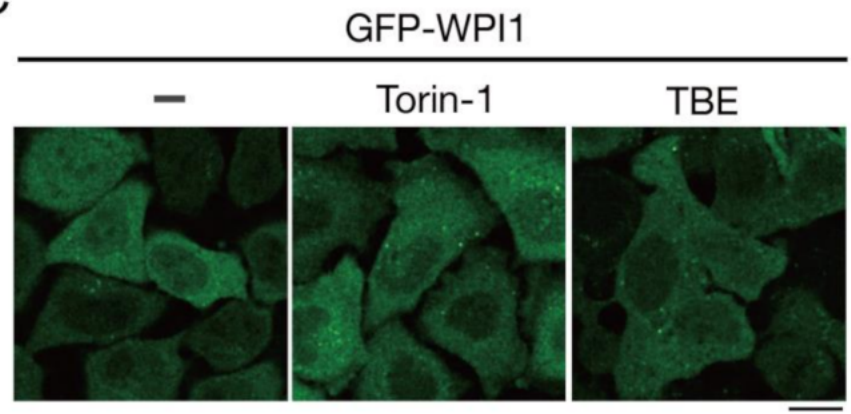

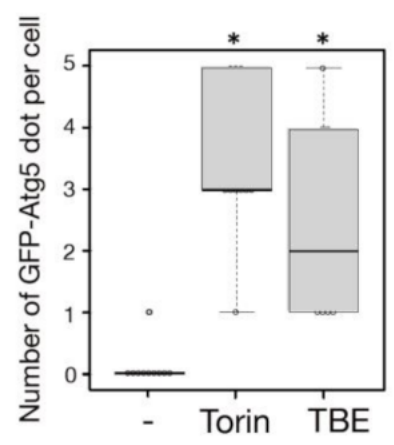
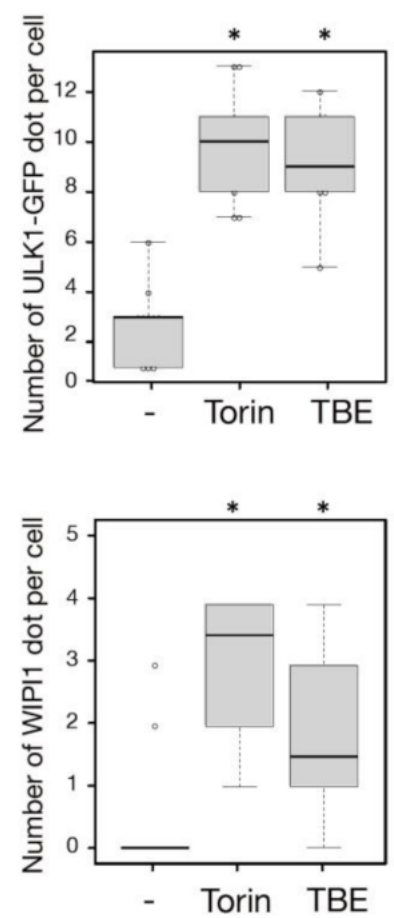

Figure 3. TBE induces autophagosome formation. HeLa cells expressing GFP-Atg5 (A), ULK1-EGFP (B), or GFP-WIPI1 (C) were treated with Torin-1 or TBE for $4 \mathrm{~h}$. The cells were observed under a confocal microscope. The number of GFP punctae over $0.56 \mu \mathrm{m}$ in diameter was counted per cell, with over 20 cells in each sample. Median: line; upper and lower quartiles: boxes; 1.5 interquartile range: whiskers. Bar: $10 \mu \mathrm{m}$. ${ }^{*}$ denotes $p<0.05$ by unpaired two-tailed Student's $t$-test.

\subsection{TBE Enhances Dephosphorylation of mTORC1 Substrates}

To investigate how TBE induced autophagy, we focused on the protein kinase mTORC1, which negatively regulates autophagy induction by alternating the phosphorylation level of proteins involved in the autophagy regulation process [13]. Transcriptional factor EB (TFEB) is a mTORC1 kinase substrate and an important regulator of autophagy/lysosome protein biogenesis [18]. Under nutrient-rich conditions, active mTORC1 phosphorylated TFEB and retained TFEB in the cytoplasm, whereas dephosphorylated TFEB under starvation conditions translocated into the nucleus. However, when the cells were treated with TBE, GFP-TFEB was localized in the nucleus even under nutrient-rich conditions (Figure 4A,B). 
A

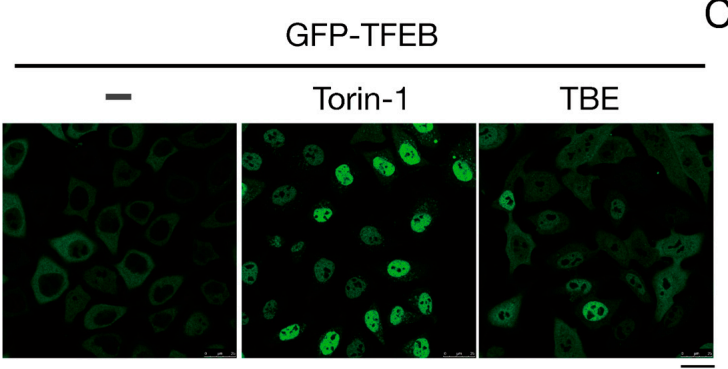

B

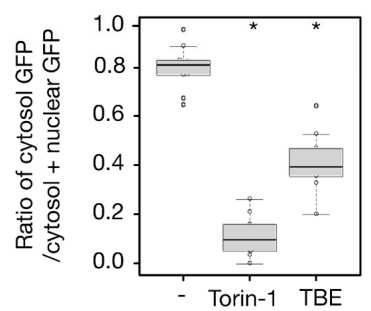

C

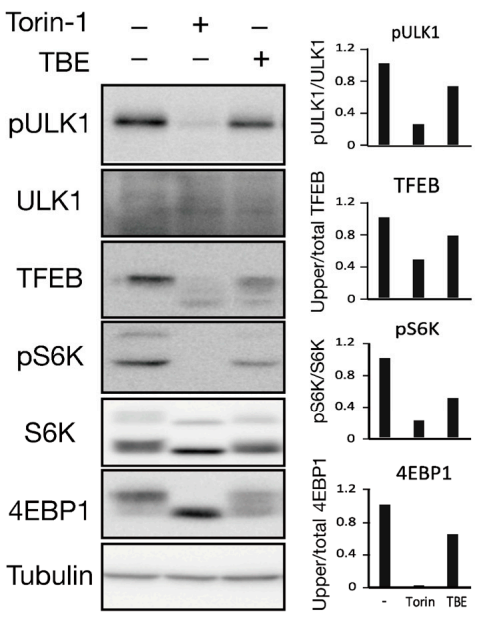

Figure 4. TBE enhances dephosphorylation of mTORC1 substrates. (A) GFP-TFEB-expressing HeLa cells were treated with or without Torin- 1 or TBE for $4 \mathrm{~h}$ and observed under a confocal microscope. Bar: $25 \mu \mathrm{m}$. (B) The graph shows the ratio of GFP intensity in a $7 \mu \mathrm{m}^{2}$ of the cytosol to the sum of GFP intensities in certain areas of the cytoplasm and nucleus. Median: line; upper and lower quartiles: boxes; 1.5 interquartile range: whiskers. ${ }^{*}$ denotes $p<0.05$ by unpaired two-tailed Student's $t$-test. (C) HeLa cells were treated with or without Torin-1 or TBE for $4 \mathrm{~h}$ and subjected to Western blotting using anti-phospho-ULK1 (Ser757), anti-ULK, anti-TFEB, anti-phospho-S6K, anti-p70 kinase, anti-4E-BP1, and anti-tubulin antibodies. The graph shows the quantification of each band intensity.

We next examined the phosphorylation levels of mTORC1 substrates by Western blotting. The band size of TFEB was shifted down in response to Torin-1, indicating that TFEB was dephosphorylated (Figure 4C). TFEB was also shifted when the cells were treated with TBE (Figure 4C).

ULK1 protein kinase was another mTORC1 kinase substrate. The serine-757 site of ULK1 was phosphorylated by active mTORC1 to suppress autophagy, while treatment with the mTORC1 inhibitor Torin-1 led to ULK1 dephosphorylation at this serine site [19]. TBE treatment also resulted in dephosphorylation of this site (Figure $4 \mathrm{C}$ ). The other major mTORC1 substrates, the ribosomal protein $\mathrm{S} 6$ kinase (S6K) and the translation initiation factor 4E-binding protein (4E-BP) [20], were also dephosphorylated in the presence of TBE (Figure 4C). Collectively, these results show that TBE suppresses mTORC1 kinase activity.

\subsection{TBE Induces Autophagy against Protein Aggregation}

In addition to bulk autophagy, which degrades nonselective cytosolic components, a wide range of specific targets are also subjected to autophagy; in this case, the process is known as selective autophagy [21]. Among these targets are aggregated proteins which are engulfed by autophagosomes and eventually degraded by a process called aggrephagy, one form of selective autophagy. In a previous study, protein aggregates were artificially induced by halting translation with puromycin treatment [22]. P62/SQSTM-1, an autophagy-related adaptor protein, is localized with protein aggregations and can be utilized as a protein aggregation marker [23]. In this study, p62-positive punctae formed after treatment with puromycin for $4 \mathrm{~h}$, representing protein aggregates (Figure 5). Eighteen hours after puromycin was washed out, most p62-positive punctae disappeared, although these punctae persisted in autophagy-defective HeLa cells [22] [Ikari et al., manuscript in preparation], indicating that their removal was dependent on aggrephagy. Torin-1 treatment accelerated this process and resulted in a reduced number of p62-positive punctae, but the effect was moderate after just a 1-h washout (Figure 5). However, TBE treatment enhanced the clearance of p62 punctae even within $1 \mathrm{hr}$ (Figure 5), indicating that TBE induces aggrephagy. 


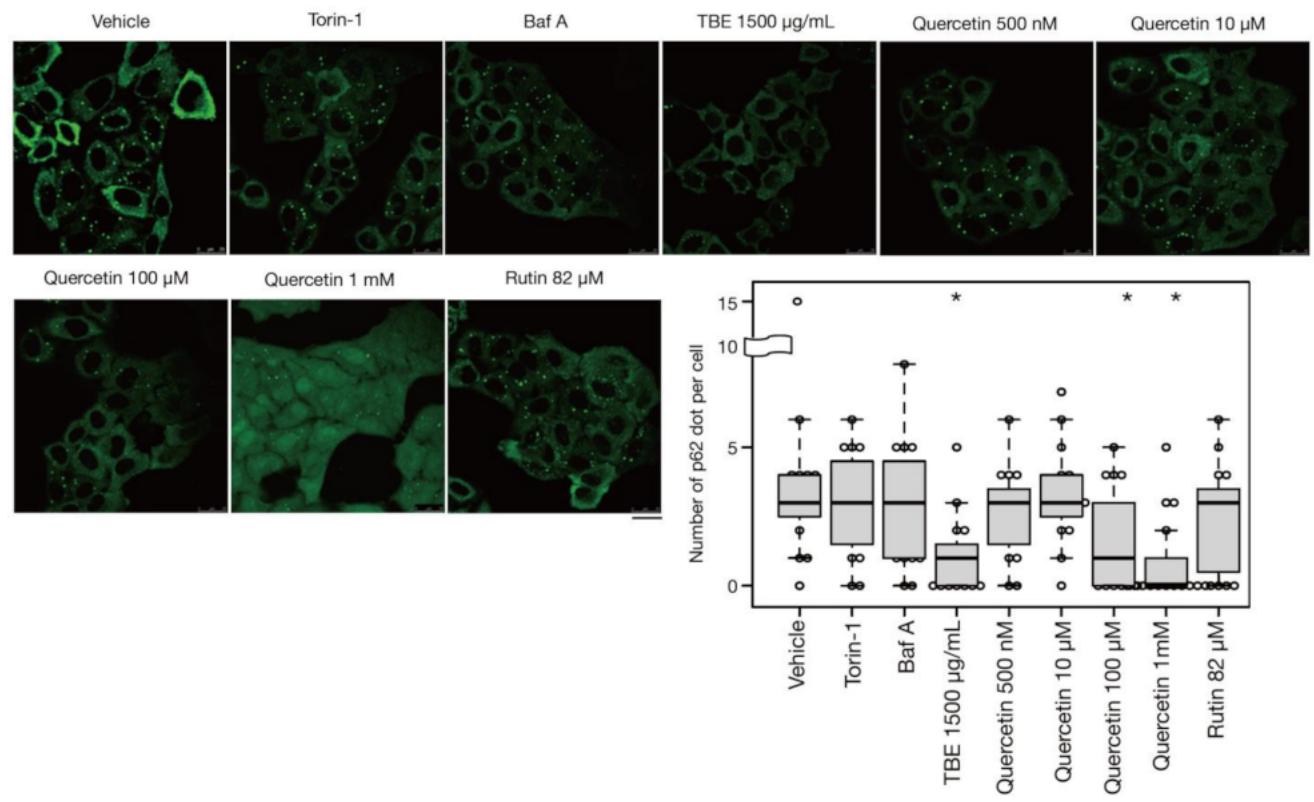

Figure 5. TBE and quercetin enhance the induction of aggrephagy. HeLa cells were treated with puromycin for $4 \mathrm{~h}$, Figure 1. Bafilomycin A1, TBE, quercetin, or rutin at the indicated concentrations for $1 \mathrm{~h}$. p62 was detected by immunostaining. The graph shows the number of p62 dots over $1.4 \mu \mathrm{m}$ in diameter per HeLa cell. Bar: $25 \mu \mathrm{m}$. Median: line; upper and lower quartiles: boxes; 1.5 interquartile range: whiskers. ${ }^{*}$ denotes $p<0.05$ by unpaired two-tailed Student's $t$-test.

\subsection{Quercetin Is Responsible for the TBE Effect on Autophagy Induction}

Rutin is a flavonoid that occurs at much higher concentrations in tartary buckwheat than in common buckwheat. We examined whether rutin could induce autophagy, but it did not do so even at a high concentration (Figure 5). Rutin consists of the flavonol quercetin and the disaccharide rutinose. A previous report showed that TBE includes a highly active rutin-degrading enzym, and, as a result, rutin is promptly degraded into quercetin in aqueous extraction $[24,25]$. We therefore examined whether quercetin affected autophagy. The TBE batch used in this study comprised $2.8 \%(w / w)$ of quercetin according to HPLC analysis (data not shown), which is equivalent to $130 \mu \mathrm{M}$ in $1500 \mu \mathrm{g} / \mathrm{mL}$ of TBE. We treated HeLa cells to determine how aggrephagy was affected by a series of quercetin concentrations. Aggrephagy was promoted by $100 \mu \mathrm{M}$ of quercetin, but not by 25 or $50 \mu \mathrm{M}$ (Figure 5). We further examined the effects of quercetin on autophagy using GFP-LC3expressing HeLa cells. When quercetin was added, the number of GFP-LC3 punctae was higher compared to vehicle control (Figure 6A). We also examined the effect of quercetin treatment on mTORC1 activity. When HeLa cells expressing GFP-TFEB were treated with quercetin, GFP-TFEB showed nuclear translocation similar to that seen following treatment with Torin-1 or TBE (Figure 6B).

\subsection{TBE and Quercetin Promote Aggrephagy in Liver Cells}

Mallory-Denk bodies are hepatocytic protein aggregates that are closely linked to the pathogenesis of alcoholic hepatitis [26]. A previous study reported that p62 aggregates accumulated in the liver of autophagy-deficient mice [27]. To study the effects of TBE and quercetin in the liver, we performed the same experiments described above using Huh7 cells, which are derived from hepatocytes. Like HeLa cells, Huh7 cells demonstrated aggrephagy induction by TBE and quercetin (Figure 6C). 
A

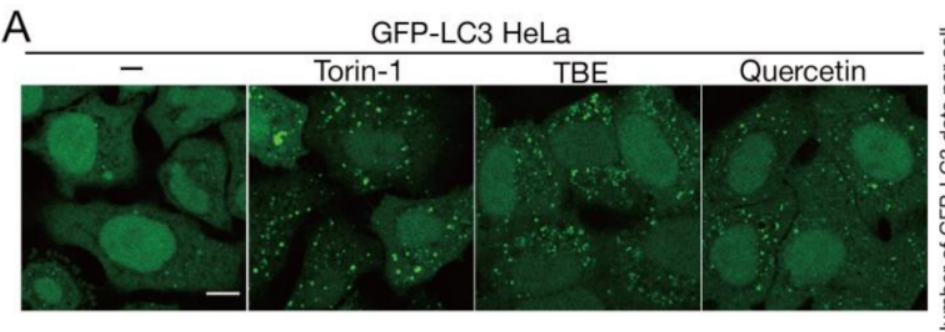

B

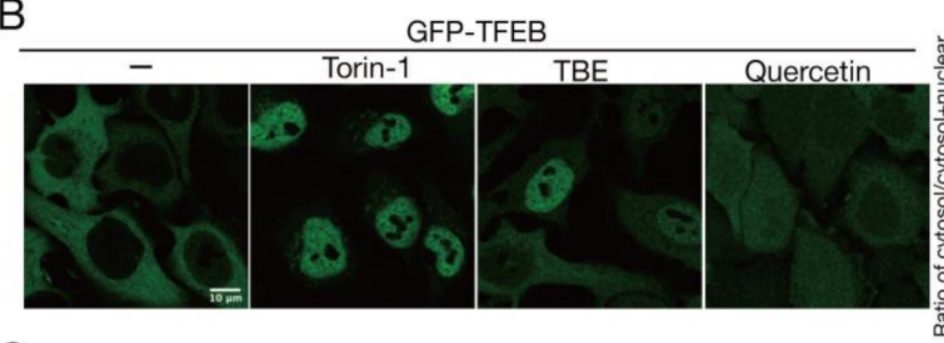

C

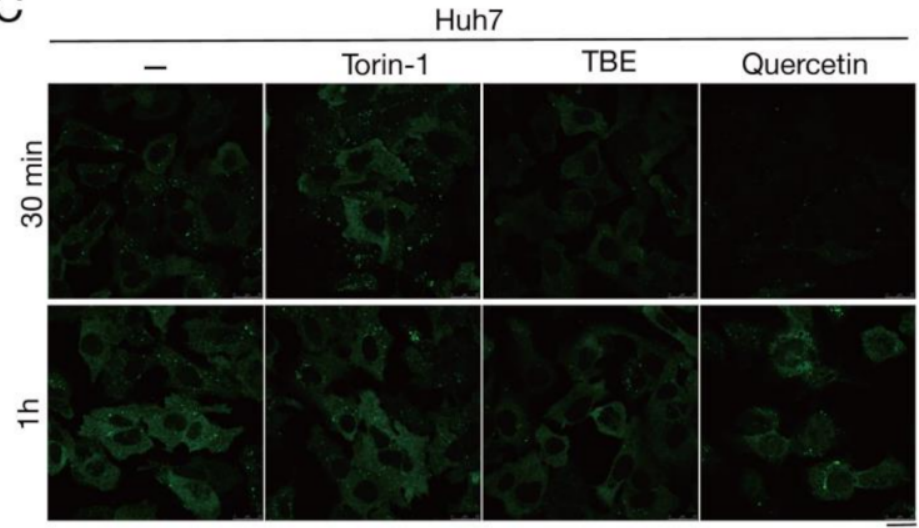

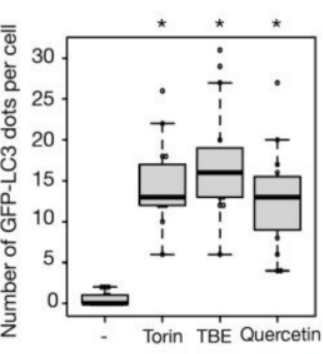

$\star *$

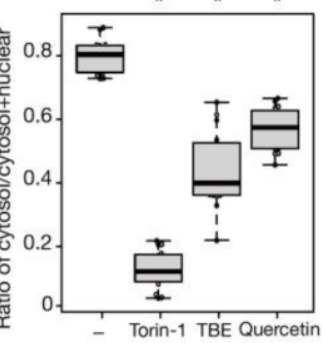

Torin-1 TBE Quercetin

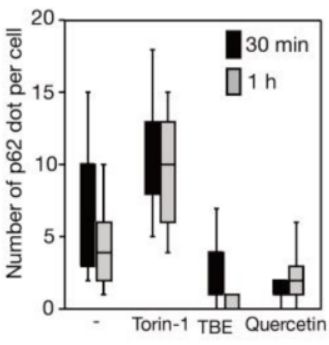

Figure 6. Quercetin enhances autophagy. (A) GFP-LC3-expressing HeLa cells were treated with or without Torin-1, TBE, or quercetin for $4 \mathrm{~h}$ under nutrient-rich conditions. The graph shows the number of GFP-LC3 punctae over $1.3 \mu \mathrm{m}$ in diameter per cell. (B) GFP-TFEB-expressing HeLa cells were treated with or without Torin-1, TBE, or quercetin for $4 \mathrm{~h}$ under nutrient-rich conditions. The graph shows the ratio of GFP intensity in a certain area of the cytosol to the sum of GFP intensities in certain areas of the cytoplasm and nucleus. (C) Huh7 cells were treated with puromycin for $4 \mathrm{~h}$, and further cultured with or without Torin-1, TBE, or quercetin for $1 \mathrm{~h}$ after washing out puromycin. Intracellular p62 was detected by immunostaining. The graph shows the number of p62 dots over $2.5 \mu \mathrm{m}$ in diameter per cell. Median: line; upper and lower quartiles: boxes; 1.5 interquartile range: whiskers. Bar: $10 \mu \mathrm{m}$ for $(\mathbf{A}, \mathbf{B}) ; 25 \mu \mathrm{m}$ for $(\mathbf{C}) .{ }^{*}$ denotes $p<0.05$ by unpaired two-tailed Student's $t$-test.

\section{Discussion}

In this study, we showed that TBE treatment induced autophagy by suppressing mTORC1 activity, and that quercetin was the ingredient that exerted this effect. We further revealed that TBE and quercetin promoted not only bulk autophagy, but also aggrephagy against aggregated proteins.

The initial phase of this project investigated the effects on autophagy of five ingredients in Maopu buckwheat liquor, and we eventually succeeded in identifying quercetin as the ingredient with potent aggrephagy-inducing ability. It remains possible that compounds in TBE other than quercetin also contribute to this effect. However, quercetin may be the primary ingredient capable of inducing autophagy on its own. Previous studies have reported that quercetin is associated with autophagy in several contexts. First, quercetin treatment induced autophagy in gastric cancer cells, causing a pronounced pro-apoptotic effect [28]. Subsequently, Klappan et al. reported that quercetin possesses a proteasome inhibitory effect that leads to mTORC1 inhibition [29]. However, in contrast with our 
findings, their study reported that quercetin treatment induced protein aggregation, mostly inside the nucleus [29]. Induction of autophagy by quercetin has been observed in several tissues and diseases, including diabetic nephropathy [30], oocytes from aged mice [31], intervertebral disc degeneration [32], myelodysplastic bone marrow [33], and human retinal pigment epithelial cells [34], while one study reported that quercetin attenuated autophagy in a rat model of traumatic brain injury [35]. Regardless of these previous studies, here we performed a comprehensive set of autophagy assays that unquestionably proved the effect of quercetin on autophagy for the first time [36]. As suggested by our study and the aforementioned reports, these effects could be mediated by mTORC1 suppression. The underlying molecular mechanism is still to be determined, but a recent report that quercetin directly binds to mTORC1 has interesting implications [37].

Both TBE and quercetin induce bulk autophagy and aggrephagy. TBE and quercetin are less effective at suppressing mTORC1 than Troin-1. Nevertheless, TBE and quercetin induce aggrephagy at an even earlier time $(1 \mathrm{hr})$ than the point at which Torin-1 exerted its limited effect on aggrephagy. Therefore, we speculate that a pathway other than that involving mTORC1 is affected by quercetin in aggrephagy, although the identity of this additional pathway is still to be determined. In the liver, the pathogenesis of alcoholic hepatitis is closely linked to the accumulation of protein aggregates called Mallory-Denk bodies [26], and the clearance of these aggregates could potentially alleviate hepatitis. In addition, protein aggregates in the liver provoke tumorigenesis [38], and aggrephagy is capable of clearing these proteins [27]. Quercetin is linked to alcohol-induced liver injury because it enhances autophagy against mitochondria (mitophagy) [39] and lipid droplets (lipophagy) [40]. Combined with these effects, the ability of TBE to enhance aggrephagy could potentially prevent these diseases. Future research to clarify this possibility is needed, especially using animal models.

Supplementary Materials: The following are available online at https://www.mdpi.com/article/ 10.3390/antiox10081217/s1, Figure S1: The effects of ethanol and TBE on cell viability.

Author Contributions: Conceptualization, T.N. and F.H.; methodology, S.-L.L.; validation, T.N., S.-L.L.; formal analysis, S.I.; investigation, S.I.; resources, Q.Y., Y.L., G.T., S.L.; writing—original draft preparation, S.I. and S.-L.L.; writing —review and editing, T.N.; project administration, T.N.; funding acquisition, Y.L. All authors have read and agreed to the published version of the manuscript.

Funding: This study was financially supported by the Hubei Provincial Key Research and Development Program (2020BBA050).

Institutional Review Board Statement: Not applicable.

Informed Consent Statement: Not applicable.

Data Availability Statement: The data presented in this study are openly available in FigShare at DOI [10.6084/m9.figshare.15057939].

Acknowledgments: The authors appreciate Kanae Noda-Shirahama for her technical contribution to the study.

Conflicts of Interest: The authors declare no conflict of interest. The sponsors had no role in the design of the study; in the collection, analyses, or interpretation of data; in the writing of the manuscript; or in the decision to publish the results.

\section{References}

1. Mizushima, N.; Yoshimori, T.; Ohsumi, Y. The Role of Atg Proteins in Autophagosome Formation. Annu. Rev. Cell Dev. Biol. 2011, 27, 107-132. [CrossRef]

2. Nakatogawa, H. Mechanisms Governing Autophagosome Biogenesis. Nat. Rev. Mol. Cell Biol. 2020, 21, 439-458. [CrossRef] [PubMed]

3. Thorburn, A. Autophagy and Disease. J. Biol. Chem. 2018, 293, 5425-5430. [CrossRef]

4. Fleming, A.; Noda, T.; Yoshimori, T.; Rubinsztein, D.C. Chemical Modulators of Autophagy as Biological Probes and Potential Therapeutics. Nat. Chem. Biol. 2011, 7, 9-17. [CrossRef] [PubMed] 
5. Wang, S.-F.; Wu, M.-Y.; Cai, C.-Z.; Li, M.; Lu, J.-H. Autophagy Modulators from Traditional Chinese Medicine: Mechanisms and Therapeutic Potentials for Cancer and Neurodegenerative Diseases. J. Ethnopharmacol. 2016, 194, 861-876. [CrossRef] [PubMed]

6. Ikari, S.; Lu, S.-L.; Hao, F.; Imai, K.; Araki, Y.; Yamamoto, Y.; Tsai, C.-Y.; Nishiyama, Y.; Shitan, N.; Yoshimori, T.; et al. StarvationInduced Autophagy via Calcium-Dependent TFEB Dephosphorylation Is Suppressed by Shigyakusan. PLoS ONE 2020, 15, e0230156. [CrossRef]

7. Jin, H.-M.; Wei, P. Anti-Fatigue Properties of Tartary Buckwheat Extracts in Mice. Int. J. Mol. Sci. 2011, 12, 4770-4780. [CrossRef]

8. Sadauskiene, I.; Liekis, A.; Bernotiene, R.; Sulinskiene, J.; Kasauskas, A.; Zekonis, G. The Effects of Buckwheat Leaf and Flower Extracts on Antioxidant Status in Mouse Organs. Oxidative Med. Cell. Longev. 2018, 2018, 1-7. [CrossRef]

9. Wang, L.; Yang, X.; Qin, P.; Shan, F.; Ren, G. Flavonoid Composition, Antibacterial and Antioxidant Properties of Tartary Buckwheat Bran Extract. Ind. Crop. Prod. 2013, 49, 312-317. [CrossRef]

10. Kimura, S.; Fujita, N.; Noda, T.; Yoshimori, T. Monitoring Autophagy in Mammalian Cultured Cells through the Dynamics of LC3. In Methods in Enzymology; Elsevier: Amsterdam, The Netherlands, 2009; Volume 452, pp. 1-12; ISBN 978-0-12-374547-7.

11. Kabeya, Y. LC3, a Mammalian Homologue of Yeast Apg8p, Is Localized in Autophagosome Membranes after Processing. EMBO J. 2000, 19, 5720-5728. [CrossRef]

12. Kimura, S.; Noda, T.; Yoshimori, T. Dissection of the Autophagosome Maturation Process by a Novel Reporter Protein, Tandem Fluorescent-Tagged LC3. Autophagy 2007, 3, 452-460. [CrossRef] [PubMed]

13. Noda, T. Regulation of Autophagy through TORC1 and MTORC1. Biomolecules 2017, 7, 52. [CrossRef]

14. Tanida, I.; Minematsu-Ikeguchi, N.; Ueno, T.; Kominami, E. Lysosomal Turnover, but Not a Cellular Level, of Endogenous LC3 Is a Marker for Autophagy. Autophagy 2005, 1, 84-91. [CrossRef] [PubMed]

15. Mizushima, N.; Yamamoto, A.; Hatano, M.; Kobayashi, Y.; Kabeya, Y.; Suzuki, K.; Tokuhisa, T.; Ohsumi, Y.; Yoshimori, T. Dissection of Autophagosome Formation Using Apg5-Deficient Mouse Embryonic Stem Cells. J. Cell Biol. 2001, 152, 657-668. [CrossRef] [PubMed]

16. Mizushima, N. The Role of the Atg1/ULK1 Complex in Autophagy Regulation. Curr. Opin. Cell Biol. 2010, 22, 132-139. [CrossRef]

17. Proikas-Cezanne, T.; Ruckerbauer, S.; Stierhof, Y.-D.; Berg, C.; Nordheim, A. Human WIPI-1 Puncta-Formation: A Novel Assay to Assess Mammalian Autophagy. FEBS Lett. 2007, 581, 3396-3404. [CrossRef]

18. Settembre, C.; Di Malta, C.; Polito, V.A.; Arencibia, M.G.; Vetrini, F.; Erdin, S.; Erdin, S.U.; Huynh, T.; Medina, D.; Colella, P.; et al. TFEB Links Autophagy to Lysosomal Biogenesis. Science 2011, 332, 1429-1433. [CrossRef]

19. Kim, J.; Kundu, M.; Viollet, B.; Guan, K.-L. AMPK and MTOR Regulate Autophagy through Direct Phosphorylation of Ulk1. Nat. Cell Biol. 2011, 13, 132-141. [CrossRef]

20. Fingar, D.C.; Richardson, C.J.; Tee, A.R.; Cheatham, L.; Tsou, C.; Blenis, J. MTOR Controls Cell Cycle Progression through Its Cell Growth Effectors S6K1 and 4E-BP1/Eukaryotic Translation Initiation Factor 4E. MCB 2004, 24, 200-216. [CrossRef]

21. Zaffagnini, G.; Martens, S. Mechanisms of Selective Autophagy. J. Mol. Biol. 2016, 428, 1714-1724. [CrossRef]

22. Kirkin, V.; Lamark, T.; Sou, Y.-S.; Bjørkøy, G.; Nunn, J.L.; Bruun, J.-A.; Shvets, E.; McEwan, D.G.; Clausen, T.H.; Wild, P.; et al. A Role for NBR1 in Autophagosomal Degradation of Ubiquitinated Substrates. Mol. Cell 2009, 33, 505-516. [CrossRef]

23. Komatsu, M.; Waguri, S.; Koike, M.; Sou, Y.; Ueno, T.; Hara, T.; Mizushima, N.; Iwata, J.; Ezaki, J.; Murata, S.; et al. Homeostatic Levels of P62 Control Cytoplasmic Inclusion Body Formation in Autophagy-Deficient Mice. Cell 2007, 131, 1149-1163. [CrossRef] [PubMed]

24. Yasuda, T.; Masaki, K.; Kashiwagi, T. An Enzyme Degrading Rutin in Tartary Buckwheat Seeds. Nippon Shokuhin Kogyo Gakkaishi 1992, 39, 994-1000. [CrossRef]

25. Zhang, Y.; Li, J.; Yuan, Y.; Gu, J.; Chen, P. Purification, characterization and partial primary structure analysis of rutin-degrading enzyme in tartary buckwheat seeds. Sheng Wu Gong Cheng Xue Bao 2017, 33, 796-807. [CrossRef]

26. French, S.; Masouminia, M.; Samadzadeh, S.; Tillman, B.; Mendoza, A.; French, B. Role of Protein Quality Control Failure in Alcoholic Hepatitis Pathogenesis. Biomolecules 2017, 7, 11. [CrossRef]

27. Komatsu, M.; Waguri, S.; Ueno, T.; Iwata, J.; Murata, S.; Tanida, I.; Ezaki, J.; Mizushima, N.; Ohsumi, Y.; Uchiyama, Y.; et al. Impairment of Starvation-Induced and Constitutive Autophagy in Atg7-Deficient Mice. J. Cell Biol. 2005, 169, 425-434. [CrossRef] [PubMed]

28. Wang, K.; Liu, R.; Li, J.; Mao, J.; Lei, Y.; Wu, J.; Zeng, J.; Zhang, T.; Wu, H.; Chen, L.; et al. Quercetin Induces Protective Autophagy in Gastric Cancer Cells: Involvement of Akt-MTOR- and Hypoxia-Induced Factor 1 $\alpha$-Mediated Signaling. Autophagy 2011, 7, 966-978. [CrossRef]

29. Klappan, A.K.; Hones, S.; Mylonas, I.; Brüning, A. Proteasome Inhibition by Quercetin Triggers Macroautophagy and Blocks MTOR Activity. Histochem. Cell Biol. 2012, 137, 25-36. [CrossRef] [PubMed]

30. Lu, Q.; Ji, X.-J.; Zhou, Y.-X.; Yao, X.-Q.; Liu, Y.-Q.; Zhang, F.; Yin, X.-X. Quercetin Inhibits the MTORC1/P70S6K SignalingMediated Renal Tubular Epithelial-Mesenchymal Transition and Renal Fibrosis in Diabetic Nephropathy. Pharmacol. Res. 2015, 99, 237-247. [CrossRef] [PubMed]

31. Cao, Y.; Zhao, H.; Wang, Z.; Zhang, C.; Bian, Y.; Liu, X.; Zhang, C.; Zhang, X.; Zhao, Y. Quercetin Promotes in Vitro Maturation of Oocytes from Humans and Aged Mice. Cell Death Dis. 2020, 11, 965. [CrossRef] [PubMed]

32. Wang, D.; He, X.; Wang, D.; Peng, P.; Xu, X.; Gao, B.; Zheng, C.; Wang, H.; Jia, H.; Shang, Q.; et al. Quercetin Suppresses Apoptosis and Attenuates Intervertebral Disc Degeneration via the SIRT1-Autophagy Pathway. Front. Cell Dev. Biol. 2020, 8 , 613006. [CrossRef] 
33. Daw, S.; Law, S. Quercetin Induces Autophagy in Myelodysplastic Bone Marrow Including Hematopoietic Stem/Progenitor Compartment. Environ. Toxicol. 2021, 36, 149-167. [CrossRef]

34. Li, D.L.; Mao, L.; Gu, Q.; Wei, F.; Gong, Y.-Y. Quercetin Protects Retina External Barrier from Oxidative Stress Injury by Promoting Autophagy. Cutan. Ocul. Toxicol. 2021, 40, 7-13. [CrossRef]

35. Du, G.; Zhao, Z.; Chen, Y.; Li, Z.; Tian, Y.; Liu, Z.; Liu, B.; Song, J. Quercetin Attenuates Neuronal Autophagy and Apoptosis in Rat Traumatic Brain Injury Model via Activation of PI3K/Akt Signaling Pathway. Neurol. Res. 2016, 38, 1012-1019. [CrossRef] [PubMed]

36. Klionsky, D.J.; Abdel-Aziz, A.K.; Abdelfatah, S.; Abdellatif, M.; Abdoli, A.; Abel, S.; Abeliovich, H.; Abildgaard, M.H.; Abudu, Y.P.; Acevedo-Arozena, A.; et al. Guidelines for the Use and Interpretation of Assays for Monitoring Autophagy (4th Edition). Autophagy 2021, 17, 1-382. [CrossRef]

37. Huang, Y.; Chen, Y.; Shaw, A.M.; Goldfine, H.; Tian, J.; Cai, J. Enhancing TFEB-Mediated Cellular Degradation Pathways by the MTORC1 Inhibitor Quercetin. Oxidative Med. Cell. Longev. 2018, 2018, 1-9. [CrossRef] [PubMed]

38. Takamura, A.; Komatsu, M.; Hara, T.; Sakamoto, A.; Kishi, C.; Waguri, S.; Eishi, Y.; Hino, O.; Tanaka, K.; Mizushima, N. Autophagy-Deficient Mice Develop Multiple Liver Tumors. Genes Dev. 2011, 25, 795-800. [CrossRef]

39. Yu, X.; Xu, Y.; Zhang, S.; Sun, J.; Liu, P.; Xiao, L.; Tang, Y.; Liu, L.; Yao, P. Quercetin Attenuates Chronic Ethanol-Induced Hepatic Mitochondrial Damage through Enhanced Mitophagy. Nutrients 2016, 8, 27. [CrossRef] [PubMed]

40. Zeng, H.; Guo, X.; Zhou, F.; Xiao, L.; Liu, J.; Jiang, C.; Xing, M.; Yao, P. Quercetin Alleviates Ethanol-Induced Liver Steatosis Associated with Improvement of Lipophagy. Food Chem. Toxicol. 2019, 125, 21-28. [CrossRef] 